\title{
Differentiating Prenatal Exposure to Methamphetamine and Alcohol versus Alcohol and Not Methamphetamine using Tensor-Based Brain Morphometry and Discriminant Analysis
}

\author{
Elizabeth R. Sowell, ${ }^{1,2}$ Alex D. Leow, ${ }^{1}$ Susan Y. Bookheimer, ${ }^{3}$ Lynne M. Smith, ${ }^{4}$ Mary J. O'Connor, ${ }^{3}$ Eric Kan, ${ }^{1,2}$ \\ Carly Rosso, ${ }^{1,2}$ Suzanne Houston, ${ }^{1,2}$ Ivo D. Dinov, ${ }^{1}$ and Paul M. Thompson ${ }^{1}$ \\ ${ }^{1}$ Laboratory of Neuro Imaging, Department of Neurology, ${ }^{2}$ Developmental Cognitive Neuroimaging Group, Department of Neurology, and ${ }^{3}$ Department of \\ Psychiatry and Biobehavioral Sciences, University of California, Los Angeles, Los Angeles, California 90095, and ${ }^{4}$ Department of Pediatrics, Los Angeles \\ Biomedical Research Institute at Harbor/University of California, Los Angeles Medical Center, Torrance, California 90502
}

Here we investigate the effects of prenatal exposure to methamphetamine (MA) on local brain volume using magnetic resonance imaging. Because many who use MA during pregnancy also use alcohol, a known teratogen, we examined whether local brain volumes differed among 61 children (ages 5-15 years), 21 with prenatal MA exposure, 18 with concomitant prenatal alcohol exposure (the MAA group), 13 with heavy prenatal alcohol but not MA exposure (ALC group), and 27 unexposed controls. Volume reductions were observed in both exposure groups relative to controls in striatal and thalamic regions bilaterally and in right prefrontal and left occipitoparietal cortices. Striatal volume reductions were more severe in the MAA group than in the ALC group, and, within the MAA group, a negative correlation between full-scale intelligence quotient (FSIQ) scores and caudate volume was observed. Limbic structures, including the anterior and posterior cingulate, the inferior frontal gyrus (IFG), and ventral and lateral temporal lobes bilaterally, were increased in volume in both exposure groups. Furthermore, cingulate and right IFG volume increases were more pronounced in the MAA than ALC group. Discriminant function analyses using local volume measurements and FSIQ were used to predict group membership, yielding factor scores that correctly classified $72 \%$ of participants in jackknife analyses. These findings suggest that striatal and limbic structures, known to be sites of neurotoxicity in adult MA abusers, may be more vulnerable to prenatal MA exposure than alcohol exposure and that more severe striatal damage is associated with more severe cognitive deficit.

\section{Introduction}

Methamphetamine (MA) abuse is a continuing problem, and data from the 2002-2004 National Surveys on Drug Use and Health indicated that 16-17 million Americans over the age of 12 years have used methamphetamine (Colliver et al., 2006). Of those, $\sim 19,000$ were pregnant women (Colliver et al., 2006). Significant advances have been made in understanding the neurotoxic effects of MA on brain structure in adults (for review, see Chang et al., 2007; Berman et al., 2008). Abuse of the drug over time causes damage to dopaminergic and sero-

\footnotetext{
Received 0ct. 5, 2009; revised Jan. 19, 2010; accepted Jan. 22, 2010.

This work was supported by National Institute of Drug Abuse Grants R21 DA15878 and R01 DA017831, National Institute of Alcoholism and Alcohol Abuse Grant U01 AA017122, and March of Dimes Grants 5FY03-12 and 6-FY2008 (E.R.S.). Additional support was provided by National Center on Research Resources, General Clinical Research Center Grant 3 M01 RR00425 (L.M.S.) and National Institutes of Health (NIH) through the NIH Roadmap for Medical Research Grant U54 RR021813 entitled Center for Computational Biology (http://nihroadmap.nih.gov/bioinformatics).

Correspondence should be addressed to Dr. Elizabeth R. Sowell, David Geffen School of Medicine at University of California, Los Angeles, Developmental Cognitive Neuroimaging Group, Laboratory of Neuro Imaging, 710 Westwood Plaza, Room 3-234, Los Angeles, CA 90095-7332. E-mail: esowell@ucla.edu.

D0I:10.1523/JNEUROSCI.4967-09.2010

Copyright $\odot 2010$ the authors $\quad 0270-6474 / 10 / 303876-10 \$ 15.00 / 0$
}

tonergic brain regions, most prominent in the basal ganglia. Limbic volume reductions have been observed in the cingulate and medial temporal lobes in MA abusers (Thompson et al., 2004). The effects of prenatal exposure to MA, when the brain is still developing, is not yet well understood.

Brain proton spectroscopy studies revealed higher creatine in the striatum and frontal lobe white matter of children with prenatal MA exposure, reflecting MA-induced changes in cellular metabolism and perhaps accelerated neuronal and glial development (Smith et al., 2001; Chang et al., 2009). Quantitative morphological analysis revealed smaller putamen, globus pallidus, and hippocampal volumes in children with prenatal MA exposure. Volume reduction in these structures correlated with poorer performance on attention and verbal memory (Chang et al., 2004). Diffusion tensor imaging (DTI) studies of children with prenatal MA exposure revealed abnormalities of frontal and parietal white matter, including increased fractional anisotropy (FA) and decreased diffusion (Cloak et al., 2009). Finally, recent work by our group revealed more diffuse brain activation in children with prenatal MA exposure during verbal learning (Lu et al., 2009). Together, the small brain imaging literature on prenatal MA exposure 
shows prominent effects in the frontostriatal network, known to be impacted from animal and human studies of adult MA abusers.

Efforts to understand specific effects of prenatal methamphetamine exposure on brain development in humans are complicated by high rates of concomitant alcohol use during pregnancy. Of pregnant MA users who participated in a study on prenatal MA exposure after giving birth, 49\% reported having used alcohol during pregnancy (Smith et al., 2006). Alcohol is a known teratogen, frequently resulting in brain and cognitive abnormalities (for review, see Riley and McGee, 2005). We evaluated the specific effects of prenatal exposure to MA on brain development by including a group of children with heavy prenatal alcohol and not MA exposure as a contrast group. Given findings from previous studies of MA exposure (Chang et al., 2004) and the known vulnerability of dopamine receptors to MA, we expected striatal volume reductions in our MA group [including those with MA and alcohol exposure (MAA)] relative to both typically developing controls and the alcohol-only contrast group. We expected cortical and white matter abnormalities to localize in frontal and parietal regions, given findings of white matter abnormalities in these regions from DTI studies (Cloak et al., 2009).

\section{Materials and Methods}

\section{Participants}

Sixty-one participants were assigned to a methamphetamine-exposed group (MAA), an alcohol-exposed group (ALC), or a nonexposed control group $(\mathrm{CON})$ based on exposure histories, which were established by an extensive interview administered to the adult guardians of minor participants. Exact quantities and patterns of maternal use of alcohol and methamphetamine are difficult to ascertain in retrospective studies, so social, medical, and/or legal records were used when available to confirm exposure histories. Of the three biological MA and/or alcohol-abusing mothers interviewed, all reported using MA and/or alcohol throughout pregnancy. The rest suffered legal and social problems as a result of their MA and/or alcohol use to the extent that their children were removed from their custody because of drug abuse in the home, neglect, or domestic violence according to reports of adoptive parents, legal, social, or medical records.

Primary recruitment mechanisms for methamphetamine-exposed participants included a rehabilitation program serving women with children born positive for methamphetamine, a social-skills group at the University of California, Los Angeles (UCLA) for children diagnosed with fetal alcohol spectrum disorders (FASDs), and word-of-mouth advertising. Of those recruited, participants were included in the methamphetamine group (MAA) if prenatal exposure to methamphetamine was sufficiently confirmed by parental or guardian report or reliable collateral report during the history interview $(n=21)$. Eighteen of the MAA children were also exposed to alcohol prenatally. Children were excluded from the MAA group if (1) they had prenatal exposure to cocaine or other opiates, (2) they were $<5$ years of age, (3) they had an intelligence quotient (IQ) of $<70$, (4) they had suffered a head injury with loss of consciousness $>20 \mathrm{~min}$, (5) they had a physical (e.g., hemiparesis), psychiatric, or developmental disability (e.g., autism) that would preclude participation, (6) they had other potential known causes of mental deficiency (e.g., chromosomal disorders), (7) significant maternal illness that has increased risk for fetal hypoxia (e.g., sickle cell disease) was reported, or (8) they had metallic implants in the body that posed a risk for magnetic resonance imaging (MRI).

Alcohol-exposed participants $(n=13)$ were recruited from the same UCLA social skills group, described above, that serves children with fetal alcohol spectrum disorders. Participants in this group met the following inclusion criteria: (1) had exposure to four or more drinks per occasion at least once per week or 14 drinks or more per week and (2) were not exposed to methamphetamine during gestation. Exclusionary criteria were the same as for the MAA group.

Details of diagnostic procedures for alcohol-related disorders within the MAA and ALC groups are described in another report (O'Connor et al., 2006). Briefly, an experienced clinician examined alcohol-exposed children using the Diagnostic Guide for Fetal Alcohol Syndrome and Related Conditions (Astley, 2004). This system uses a four-digit diagnostic code reflecting the magnitude of expression of four key diagnostic features of fetal alcohol syndrome (FAS): (1) growth deficiency; (2) the FAS facial phenotype, including short palpebral fissures, flat philtrum, and thin upper lip; (3) CNS dysfunction; and (4) gestational alcohol exposure. All of the children with FASD diagnoses had histories of heavy prenatal alcohol exposure confirmed by maternal report, reliable collateral report, and/or medical or legal records. Using this four-digit code, children were diagnosed with FAS, partial FAS (PFAS), alcohol-related neurodevelopmental disorder (ARND), or sentinal features. Recent studies have shown brain morphological abnormalities to be more severe in children with the facial phenotype to obtain a diagnosis of FAS or PFAS relative to children with alcohol-related disorders but not facial dysmorphology (Astley et al., 2009). Five subjects in the ALC group and four subjects in the MAA group were diagnosed with FAS or PFAS, and the rest had ARND or sentinal features.

Nonexposed control participants $(n=27)$ were recruited from the same Los Angeles communities as both the MAA and ALC exposed participants, using bulk mail advertisements, magazine advertisements, and flyers distributed to local libraries. Participants were excluded from the CON group if they had exposure to more than two drinks on any occasion or an average of one drink or more per week. Other exclusionary criteria were exactly the same as for the other two participant groups.

\section{Procedures}

All participants and their parents gave informed assent/consent according to procedures approved by the UCLA Institutional Review Board.

\section{Image acquisition}

High-resolution $\mathrm{T}_{1}$-weighted sagittal volumes were collected from a 1.5 T Siemens Sonata scanner (repetition time, $1900 \mathrm{~ms}$; echo time, $4.38 \mathrm{~ms}$; flip angle, $15^{\circ}$; matrix size, $256 \times 256 \times 160$; field of view, $256 \mathrm{~mm}$; voxel size, $1 \times 1 \times 1 \mathrm{~mm}$; acquisition time, $8 \mathrm{~min} 8 \mathrm{~s}$ ). Two to four acquisitions were acquired for each subject. Raters blind to subject age, sex, and diagnosis evaluated image quality, and data from at least two acquisitions were averaged to enhance signal-to-noise ratio (SNR).

\section{Neurocognitive evaluations}

Children underwent extensive individual neuropsychological testing. Included among tests administered was an abbreviated version of the Wechsler Intelligence Scale for Children, Fourth Edition (WISC-IV) (Wechsler, 2003). A prorated full-scale intelligence quotient (FSIQ) was derived from the prorated verbal comprehension index (VCI) and perceptual reasoning index (PRI). This method is described in the WISC-IV manual, and there is evidence that prorated VCI and PRI are each highly correlated ( $r$ values $\geq 0.90$ ) with actual index scores (Glass et al., 2008). FSIQ was not available for two of the controls subjects (not enough subtests were administered as a result of time restrictions, and one 5-year-old was too young for the WISC-IV) and one of the ALC subjects (who was unable to tolerate testing). Socioeconomic status was estimated based on annual family income obtained in a parent interview.

\section{Image analysis}

Tensor-based morphometry overview. MRI data were analyzed with tensor-based morphometry (TBM), which allows investigations of regional differences in the volumes of brain substructures by globally aligning all brain images into a common brain template before applying localized deformations to adjust each subject's anatomy to match the global group-averaged template. Detailed methods have been described previously (Leow et al., 2005, 2007). These methods were developed in-house and have been extensively tested and validated (Yanovsky et al., 2009). They have been used previously to study normal brain development (Hua et al., 2009), brain dysmorphology in fragile X syndrome (Lee et al., 2007), Williams syndrome (Chiang et al., 2007), autism (Brun et al., 2009), Alzheimer's disease and mild cognitive impairment (Hua et al., 2008b; Leow et al., 2009), and childhood-onset schizophrenia (Gogtay et al., 2008). Briefly, our implementation of TBM involves (1) nonlinearly 
deforming all images by a mutual information (MI)-based inverseconsistent elastic registration algorithm to match a preselected brain template, and (2) the three-dimensional Jacobian determinant maps of the deformation fields (mathematically defined by computing the determinant of the pointwise Jacobian operator applied to these deformations) are used to gauge the local volume differences between individual images and the template. The determinant maps can be statistically analyzed on a voxel level to identify group differences in brain structure, such as localized atrophy or tissue excess.

The MI-based inverse-consistent elastic registration algorithm uses mutual information as the image similarity measure (Wells et al., 1996) and uses an elastic regularization (computationally realized in the Fourier domain) on the deformation field. A multi-resolution scheme is used to successively compute coarse-to-fine deformations using fast Fourier transforms (FFTs) at increasing spatial resolutions. Numerical convergence is checked every 20 iterations and is defined as the point at which MI failed to increase by 0.001 after the previous iterations. Three hundred iterations are computed at each FFT resolution before increasing the resolution by a factor of 2 in each dimension (with the time step decreased to $1 / 10$ of its previous value).

Image preprocessing. The MR images for each individual go through a series of automated and manual processes. First, multiple images acquired for each subject are averaged to improve the SNR. Second, extra non-brain tissues (i.e., scalp, orbits, brainstem) are automatically removed from the image (Shattuck and Leahy, 2002). Manual edits by expert image analysts were then made to remove any errors made by the automated brain extraction tool. The skull-stripped images are then run through an automated intensity-correction program to correct any inhomogeneity artifacts introduced by the magnet (Sled et al., 1998).

Generation of a mean anatomical template. Each individual's preprocessed image data was then affinely coregistered (nine-parameter transformation) to a high-resolution MRI brain-only image volume of one representative subject. This step adjusts for individual differences in global brain scale and head alignment, and a single subject's brain may be better than making a mean template from numerous subjects, because it has sharp high-contrast edges, which helps to improve the registration of boundaries (Chiang et al., 2007). Next, a minimal deformation target (MDT), or group mean template, was constructed. This has been advocated in previous studies to reduce bias and improve statistical power (Kochunov et al., 2001; Hua et al., 2008a). To create the MDT, each subject's nine-parameter registered image is then nonlinearly registered to an average brain template or atlas comprising 102 individuals from combined developmental imaging studies conducted by the Developmental Cognitive Neuroimaging Group at UCLA, including all typically developing children and adolescents, all subjects with prenatal exposure to drugs of abuse, and all 61 subjects in the present study. We chose to use the larger sample of all subjects in our database rather than a studyspecific MDT because it may be more representative of the various neurodevelopmental studies in our group and in the larger developmental neuroimaging community. Following the steps in the study by Hua et al. (2008), the MDT was constructed by matching one three-dimensional image to the other using our MI-based inverse-consistent algorithm, followed by applying the inverse of the mean displacement field [i.e., inverse geometric centering of the corresponding displacement fields (Kochunov et al., 2002)] from all subjects to the average 102 averagebrain template.

Individual nonlinear registration to MDT. To localize differences in brain structure, each individual image is then compared with this MDT by further nonlinearly deforming the anatomy of each individual image to match that of this MDT. Last, the Jacobian operator was applied to the deformation fields, whose voxelwise determinants (the Jacobian maps) thus represent the local expansion and contraction factors required to deform the individual's anatomy to the MDT. These maps represent localized percentage change, with positive percentages representing tissue expansion and negative percentages showing tissue contraction. These Jacobian volume deformation maps can then be used to evaluate group differences in voxel-based whole-brain analyses.
Whole-brain statistical analysis

We used the computational statistics libraries provided by the Statistics Online Computational Resource (www.SOCR.ucla.edu) to conduct voxelwise multiple regression analyses throughout the entire brain, in which group membership was used to predict Jacobian values at each voxel (Che et al., 2009). These analyses yielded statistical $p$ value maps $(p<$ 0.05 ) reflecting the extent to which local volume differed across the three groups. Permutation testing (Bullmore et al., 1999) was used to correct for multiple comparisons. In these analyses, subjects were randomly assigned to groups (of the same sample sizes as the original groupings), and statistical maps for 1000 permutations were generated using the same multiple regression analyses used in the original groupings. The percentage of significant $(p<0.05)$ voxels within the brain in the 1000 random groupings was compared with the percentage of significant voxels in the real group analyses. Corrected $p$ values for the observed group difference were created by counting the number of random permutations whose suprathreshold voxels within the entire brain met or exceeded that in the original groupings. This approach has been used in multiple studies (Gogtay et al., 2008; Leow et al., 2009).

To investigate how the three groups contributed to the significant group analyses described above, follow-up tests were conducted using three two-sample $t$ tests (CON vs ALC, CON vs MAA, and ALC vs MAA) using a mask of all significant voxels in the three-group multiple regression analyses. Permutation analyses were conducted to estimate corrected $p$ values for each contrast.

Although there were no statistically significant differences in the gender distribution across the three groups, the proportion of boys was higher in the CON than the MAA or ALC groups. Thus, separate statistical analyses were conducted using gender as a nuisance covariate. Furthermore, we ran separate analyses excluding the three subjects in the MAA group who did not have concomitant alcohol exposure to ensure that children with only MA exposure were not biasing the results.

\section{Correlations with full-scale intelligence quotient}

We extracted percentage volume change estimates from each individual's Jacobian map at each of 14 locations throughout the brain. All regions selected statistically significantly differentiated groups in whole-brain three-group TBM analyses and included left and right thalamus, left and right caudate nucleus, left and right supracalcarine cortex (posterior cingulate region), right posterior inferior frontal gyrus, right superior parietal lobe, left and right temporal fusiform cortex, left occipital fusiform cortex, right parahippocampal gyrus, and left periventricular callosal white matter [see spatial coordinates in ICBM (for International Consortium for Brain Mapping) space in Table 1]. Correlations between brain Jacobian volume measures for each of the 14 anatomical points and each subject's FSIQ score were conducted, and multiple regression analyses were used to examine group $\times$ FSIQ interactions in predicting Jacobian measures.

\section{Factor analysis}

The same anatomical regions of interest (ROIs) described for the FSIQ anatomical correlations as well as FSIQ were entered into a classical discriminant function analysis (DFA) (Systat version 12) and used to model the best linear equation among these variables to predict group membership. Factor loadings above 0.3 were considered important for interpretation purposes, and results with and without jackknifed classification are reported. Similar methods have been used in other investigations of the utility of linear combinations of variables in diagnosis of disease states (Ratei et al., 2007).

\section{Results \\ Demographics}

Demographic descriptors and behavioral performance on FSIQ measures are reported in Table 2. Groups did not differ from each other in age, gender distribution, handedness, or socioeconomic status as estimated from family annual income. The groups differed in FSIQ $\left(F_{(2,55)}=9.08, p<0.001\right)$, with the CON group scoring significantly higher than the ALC and MAA groups, but 
Table 1. Jacobian determinant regions of interest: anatomical name, ICBM-305 coordinates, hemisphere, location of graph in Figure 1, Jacobian determinant correlation with FSIQ, and factor loadings from the discriminant function analyses

\begin{tabular}{|c|c|c|c|c|c|c|}
\hline $\begin{array}{l}\text { Jacobian determinant regions of } \\
\text { interest anatomical structure }\end{array}$ & $\begin{array}{l}\text { ICBM-305 } x, y, z \\
\text { coordinates }\end{array}$ & Hemisphere & Figure 1 location & $\begin{array}{l}\text { Jacobian determinant correlation } \\
\text { with FSIQ all groups combined }\end{array}$ & Loading factor 1 & Loading factor 2 \\
\hline Superior parietal lobule & $107,79,139$ & $\mathrm{R}^{a}$ & $A$ & $r=-0.004, p=0.975$ & 0.056 & -0.445 \\
\hline Supracalcarine cortex & $95,41,93$ & $\mathrm{R}$ & $B$ & $r=0.296, p=0.024$ & 0.204 & 0.368 \\
\hline Supracalcarine cortex & $85,63,94$ & $\mathrm{~L}$ & $C$ & $r=-0.198, p=0.136$ & -0.728 & 0.192 \\
\hline Thalamus & $75,101,84$ & $L$ & $D$ & $r=0.198, p=0.137$ & 0.404 & -0.481 \\
\hline Midcingulate & $98,110,122$ & $\mathrm{R}^{a}$ & $E$ & $r=0.006, p=0.963$ & 0.213 & 0.504 \\
\hline Thalamus & $101,105,86$ & $\mathrm{R}$ & $F$ & $r=0.331, p=0.011$ & 0.058 & 0.499 \\
\hline Caudate & $75,119,94$ & $\mathrm{~L}$ & $G$ & $r=0.120, p=0.371^{b}$ & 0.173 & 0.300 \\
\hline Caudate & $109,122,94$ & $\mathrm{R}$ & $H$ & $r=0.127, p=0.341$ & -0.335 & -0.424 \\
\hline Posterior inferior frontal gyrus & $154,116,92$ & $\mathrm{R}$ & I & $r=-0.057, p=0.669$ & -0.438 & -0.362 \\
\hline Temporal fusiform cortex & $127,108,44$ & $\mathrm{R}$ & $J$ & $r=-0.225, p=0.089$ & 0.190 & -0.063 \\
\hline Temporal fusiform cortex & $49,116,45$ & $\mathrm{~L}$ & $K$ & $r=-0.322, p=0.014$ & -0.209 & -0.224 \\
\hline Occipital fusiform gyrus & $64,43,67$ & $\mathrm{~L}$ & Not shown & $r=0.275, p=0.036$ & 0.355 & -0.314 \\
\hline Periventricular white matter & $54,86,82$ & $\mathrm{~L}$ & Not shown & $r=-0.088, p=0.510$ & -0.346 & -0.625 \\
\hline Parahippocampal gyrus & $116,87,66$ & $\mathrm{R}$ & Not shown & $r=0.096, p=0.472$ & -0.231 & -0.374 \\
\hline FSIQ in factor analyses & & & & & 0.420 & -0.150 \\
\hline
\end{tabular}

L, Left; $R$, right.

${ }^{a}$ Displayed as the left hemisphere in figures.

${ }^{b}$ Significant group $\times$ FSIQ interaction.

Table 2. Demographics for each group

\begin{tabular}{|c|c|c|c|c|}
\hline & MAA $(n=21)$ & $\operatorname{ALC}(n=13)$ & $\operatorname{CON}(n=27)$ & Group differences \\
\hline Age & $9.66(1.85)$ & 11.15 (2.34) & $10.15(2.90)$ & None \\
\hline Female/male & $9 / 12$ & $5 / 8$ & $16 / 11$ & None \\
\hline Handedness [right (100) to left $(-100)]$ & $60.45(47.99)$ & $55.83(59.76)$ & $70.63(28.15)$ & None \\
\hline Family annual income & $7.714(1.90)$ & $6.77(2.42)$ & $6.96(2.72)$ & None \\
\hline FSIQ & $97.81(12.77), n=21$ & $89.50(13.53), n=12$ & $110.32(16.73), n=25$ & $\begin{array}{c}F_{(2,55)}=9.08, p<0.001 \\
\quad \text { CON }>\text { ALC }, t=-4.05 \\
p<0.001 ; \text { CON }>\text { MAA } \\
t=2.87, p=0.006\end{array}$ \\
\hline
\end{tabular}

Values expressed as mean (SD). Family annual income is a categorical variable: $1,<\$ 5000 ; 2, \$ 5000-9999 ; 3, \$ 10,000-19,999 ; 4, \$ 20,000-29,999 ; 5, \$ 30,000-39,999 ; 6, \$ 40,000-49,999 ; 7, \$ 50,000-74,999 ; 8, \$ 75,000-100,000 ;$ $9,>\$ 100,000$.

the MAA and ALC groups did not significantly differ from each other.

\section{Three-group analyses}

Figure 1 maps regions of the brain that were significantly $(p<$ 0.05, uncorrected) different between the three groups determined using multiple regression analysis. Numerous brain regions are affected in individuals with prenatal drug exposure, including the striatum (as predicted), thalamus, anterior and posterior cingulate, medial and inferior temporal, dorsal and ventral frontal and parietal cortices (as predicted), and cerebellar and posterior callosal white matter. These effects were predominantly bilateral in all cortical and subcortical regions, with two exceptions: left but not right hemisphere occipital cortex and right but not left hemisphere frontal pole distinguished groups. Scatter plots of Jacobian volume measurements for each subject from 12 of the 14 regions of interest (regions that significantly differentiated groups in the statistical maps) are shown in Figure 1. Results from whole-brain permutation analyses suggest that the number of significant voxels in the three-group analyses (at the $p=0.05$ level) were not likely observed by chance (permutation $p$ value $<0.001$ )

Two separate multiple regression analyses were conducted, one using group and gender (as a nuisance covariate) to predict regional volumes and another excluding the three subjects in the MAA group who did not have concomitant alcohol exposure. The results did not substantially differ from the three-group analyses described above.

\section{Follow-up analyses}

Figures 2 and 3 show average volume differences as a percentage of volume reduction in the MAA group relative to controls (Fig. 2) and the ALC group relative to controls (Fig. 3). The regional pattern of volume increases (yellow-orange-red) and volume decreases (shades of blue and purple) in both groups relative to controls is similar in the regions that are increased (or decreased) in volume and tended to be increased (or decreased) in both exposed groups relative to controls, although to lesser or greater intensities and spatial extents. Maximum volume reductions are as high as $32 \%$ in the ALC group relative to controls. Volume increases were as high as $27 \%$ in the MAA group relative to controls and as high as $23 \%$ in the ALC group relative to controls. The three pairwise comparisons (CON vs ALC, CON vs MAA, and MAA vs ALC) are simultaneously mapped in Figure 4, showing regions of statistically significant volume differences between groups in regions in which the three-group analyses were statistically significant (from Fig. 1). In this figure, all regions in color were statistically significant in one or more of the two-group statistical analyses for volume increases and volume decreases. Subcortically, thalamic and striatal structures are reduced in volume bilaterally in both groups relative to controls as are left parieto-occipital and right anterior prefrontal cortices. On average, volume reductions in exposed children in these regions are on the order of $10-15 \%$ (Fig. 4 , shades of blue and purple), but local variation in these percentages are observed (as shown in the scatter plots in Fig. 1). Limbic cortices of the anterior and posterior cingulate, ventral and medial temporal lobes, and bilateral 
perisylvian cortices are increased in volume bilaterally in both groups relative to controls, an average of $\sim 10-15 \%$ averaged across all voxels (Fig. 4, orange, yellow, and red). It should be noted that the contrast comparing ALC with CON groups was less powerful than the contrast comparing the MAA with CON groups, because there were fewer subjects in the ALC than the MAA group. Thus, the apparent larger extent of significant positive and negative effects in the MAA subjects relative to controls than the ALC subjects relative to controls may be attributable to the increased statistical power. Wholebrain permutation analyses suggested that the number of significant voxels in both the MAA versus CON and ALC versus CON contrasts were not likely observed by chance (permutation $p$ value $<0.001$ for both contrasts).

Direct statistical comparison of the two exposed groups are also shown in Figure 4. To interpret MAA versus ALC differences, we first examined the direction of group difference in the ALC or MAA versus CON contrast and then determined which exposed group was more deviant from the controls (in volume reduction or increase), who were considered "baseline normal" for these purposes (centered on 0 in the graphs shown in Fig. 4). Volume reductions in the striatum, although observed in both groups, appear to be more severe in the MAA group (i.e., MAA volumes significantly smaller than ALC volumes by 10\%) (Fig. 4, dark blue). Volume increases were observed in both exposed groups relative to controls in anterior and posterior cingulate regions and in left and right perisylvian cortices, and these volume increases are more pronounced in the MAA than ALC group (Fig. 4, yellow; MAA volume significantly larger than ALC and CON volumes by as much as $\sim 15 \%)$. Although prominent volume increases were observed in both groups in inferolateral and some medial temporal lobe regions, the exposed groups did not significantly differ from each other in these regions. Results from permutation analyses for the direct comparison between MAA and ALC groups were not significant during permutation correction for multiple comparisons, perhaps because of the smaller samples in the exposed groups relative to the comparisons between each exposed group versus the unexposed controls.

\section{Classical discriminant function analyses}

Classical DFA of the 14 ROIs and FSIQ revealed two factor scores that discriminated the three groups with overall $72 \%$ accuracy (67\% accurate for the ALC group, 72\% accurate for the CON group, and $76 \%$ accuracy for the MAA group) using jackknife procedures (Fig. 5). Factor loadings (canonical discriminant functions standardized) on each of the 14 brain and FSIQ predictors are shown in Table 1. The first factor seems most important in determining whether or not an individual was exposed to drugs prenatally, and the second factor differentiates MAA from ALC groups. Qualitative assessment of standardized discriminant function scores for factor 1 suggest that volumes of the left supracalcarine and occipital fusiform cortex and FSIQ produced the best linear equation to discriminate exposure groups from controls. All of these measures loaded more heavily on fac- tor 1 than factor 2. Loadings for factor 2, discriminating MAA from ALC groups, relied most heavily on right superior parietal lobe, right medial prefrontal, left caudate, and right thalamus. All of these regions loaded with factor scores above 0.3 (or below -0.3 ) and were much higher on factor 2 than factor 1 .

\section{Brain volumes and FSIQ}

Exploratory analyses for simple correlations between FSIQ and Jacobian-based volume measurements for each of the 14 ROIs shown in Table 1 revealed significant correlations for several ROIs (uncorrected). Left and right occipital (supracalcarine and fusiform) Jacobian volumes were positively correlated with FSIQ such that individuals with lower volumes had lower IQ scores (left, $r=0.275, p=0.036$; right, $r=0.334, p=0.018$ ). Volume reductions in the right thalamus were also associated with reductions in FSIQ $(r=0.296, p=0.024)$. These findings make sense in light of volume decreases observed in these regions in both exposure groups relative to controls and suggest that the greater the volume reduction, the lower the intellectual ability. Left inferior temporal fusiform regions were significantly negatively correlated with FSIQ such that individuals with higher volumes had lower FSIQ scores $(r=-0.322, p=0.014)$. These results also make sense in that volume increases in these inferotemporal regions are observed in both exposed groups relative to controls and suggest that greater volume increases are associated with greater decrements in FSIQ. Although the effects for each brain region that correlated with FSIQ were in the predicted direction, it should be noted that none of these effects remained significant when group membership was used as an additional predictor. This suggests that relationships between brain volumes and FSIQ are mediated by group differences in both brain and FSIQ measures. In other words, individuals with prenatal exposure to drugs of abuse are likely to have lower FSIQ and lower volumes in the thalamus and medial occipital regions than individuals without significant prenatal drug exposure histories. Group $\times$ score interactions for these measures were significant in predicting the left caudate Jacobian-based volume measurement $(F=3.38, p=$ 0.042 ) (Fig. 5) such that, within the MAA group, smaller volumes were associated with lower FSIQ scores. In contrast to the MAA 


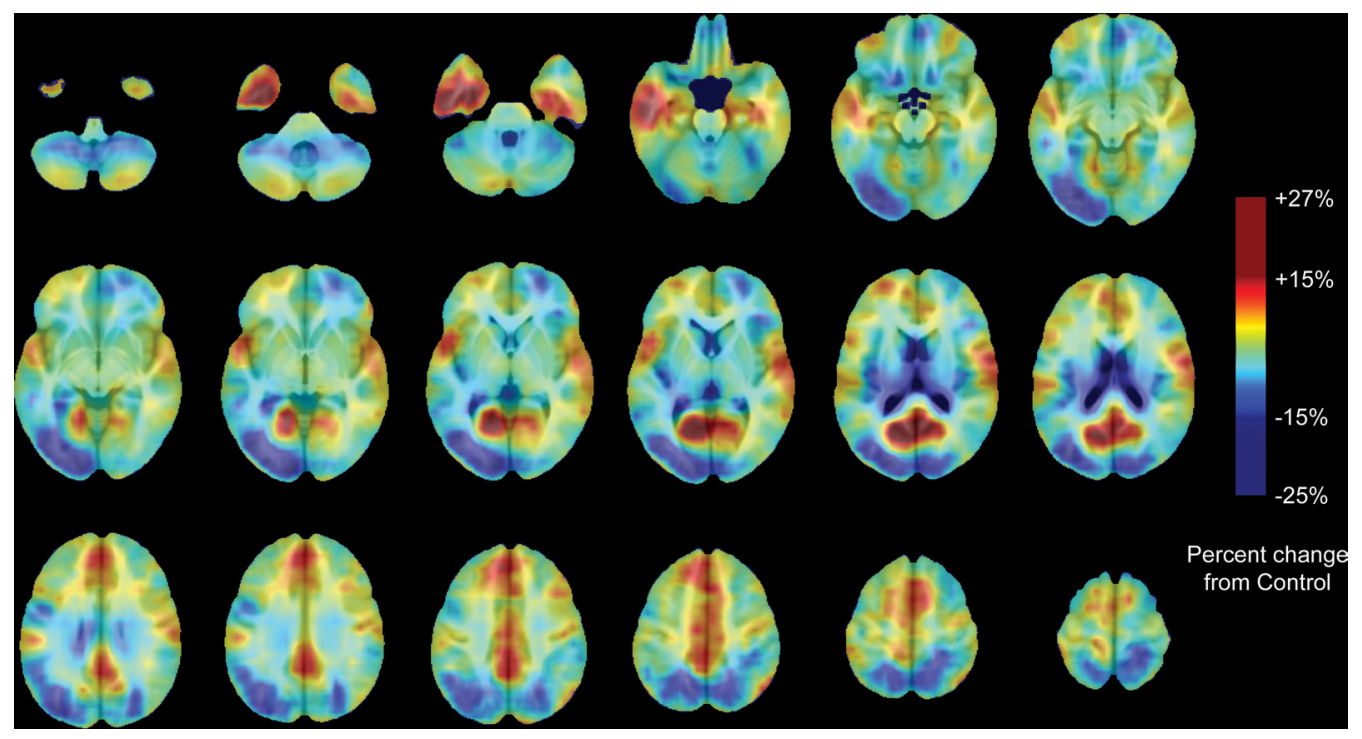

Figure 2. MAA percentage difference from average CON Jacobian values. Maps are color coded according to the color bar in which volume increases relative to controls are shown in shades of red, and volume decreases in the MAA relative to CON groups are shown in shades of blue.

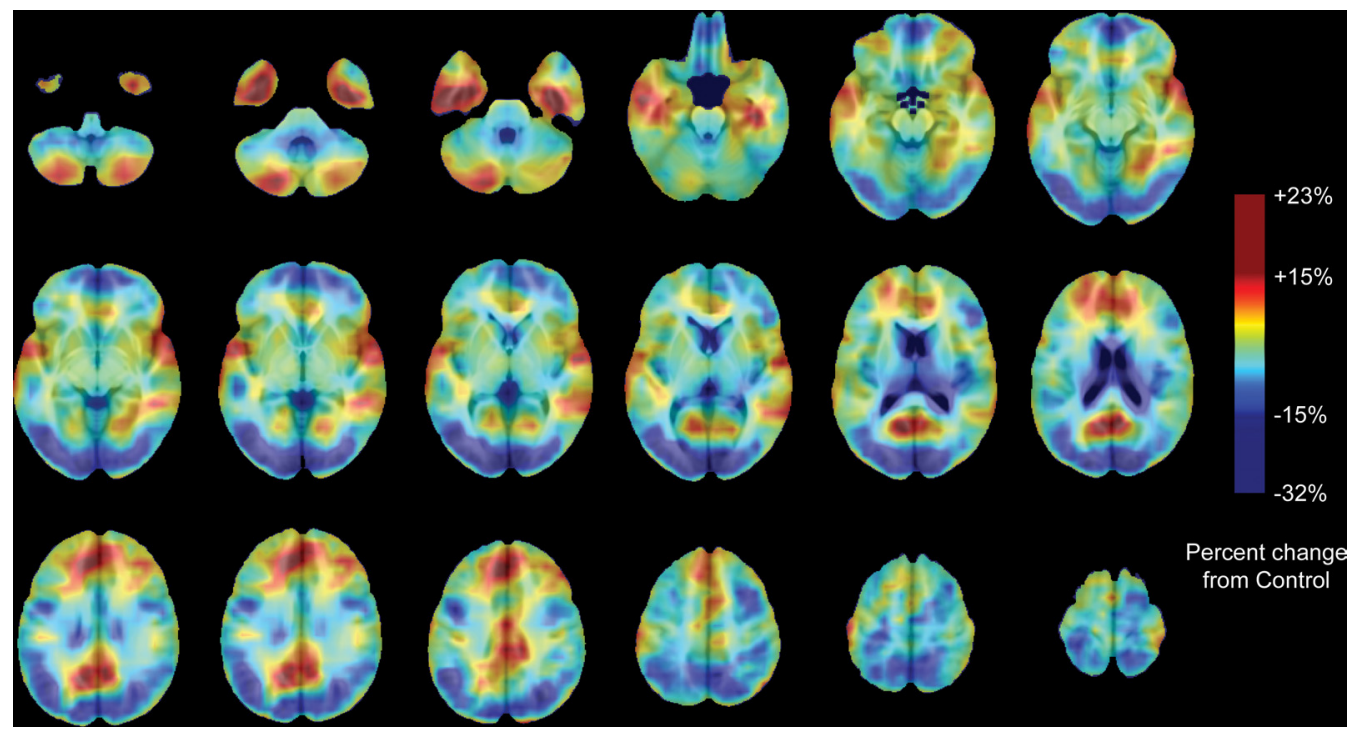

Figure 3. ALC percentage difference from average CON Jacobian values. Maps are color coded according to the color bar in which volume increases relative to controls are shown in shades of red, and volume decreases in the ALC relative to CON groups are shown in shades of blue.

group, for children in the ALC and CON groups, smaller caudate volumes were associated with higher FSIQ scores.

\section{Discussion}

We have long known that the deleterious effects of prenatal exposure to alcohol on brain morphology endure through childhood and adolescence (Archibald et al., 2001; Bookstein et al., 2001; Autti-Räm ö et al., 2002; Bookstein et al., 2002; Sowell et al., 2002, 2007) and that many women who abuse methamphetamine also abuse alcohol, but here we show for the first time that brain morphology is affected in children with prenatal MA exposure above and beyond the effects of alcohol exposure alone. Overall, the regional pattern of brain dysmorphology was similar in the two exposure groups relative to the unexposed controls. That is, regions that were reduced in the ALC group also tended to be reduced in the MAA group. Similar brain systems appear to be affected in similar ways whether the prenatal exposure is to alcohol and not MA, or alcohol and MA. Generally, subcortical structures of the striatum and thalamus, as well as more lateral cortical regions of the temporoparietal lobes bilaterally and the frontal pole in the right hemisphere, are reduced in volume in the exposed relative to control groups. The etiology of volume reductions in these regions is not entirely clear from these structural brain imaging investigations. There is evidence that MA neurotoxicity to brain structures high in monoaminergic receptors (i.e., the striatum) results in agenesis of mature axons and axon terminals (McCann and Ricaurte, 2004), but the impact of MA on developing neural systems likely has a much more pronounced effect. This is because monoaminergic circuits project widely in the brain and develop early in ontogeny, increasing the likelihood that non-aminergic neural networks could also be impacted (for review, see Frost and Cadet, 2000). Similar mechanisms could be implicated in prenatal alcohol exposure, although different brain neurotransmitter circuits may be targeted (for re- 


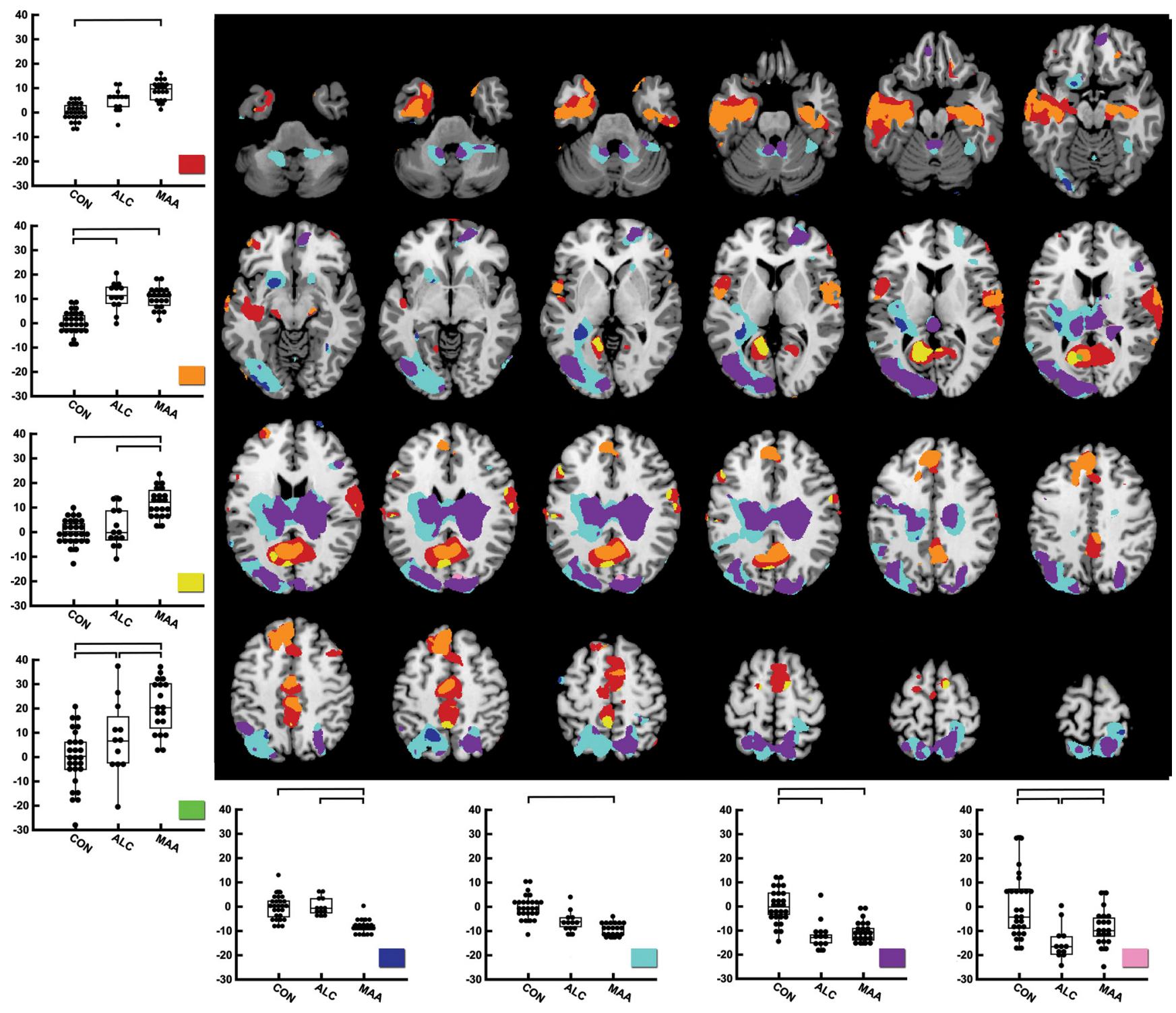

Figure 4. Follow-up test statistical binarized $p$ maps at a threshold of $p=0.05$ (uncorrected) representing the following contrast categories between groups: (1) CON $<$ MAA (red), (2) CON $<$ MAA and CON $<$ ALC (orange), (3) CON $<$ MAA and ALC $<$ MAA (yellow), (4) CON $<$ ALC, CON $<$ MAA, and ALC $<$ MAA (green), (5) CON $>$ MAA and ALC $>$ MAA (dark blue), (6) CON $>$ MAA (light blue), (7) CON $>$ ALC and CON $>$ MAA (purple), and (8) CON $>A L C, C O N>M A A$, and ALC $<$ MAA (light pink). Regions of interest were created by binarizing statistical maps for all voxels within the brain that were consistent with the color-coded contrast categories, and the average Jacobian determinant of all voxels within each of the eight regions of interest for each subject was calculated. The average Jacobian determinant value [y-axis; percentage growth or shrinkage relative to the control group (centered on 0$)$ ] for each subject for each contrast ROl is plotted by group membership (x-axis).

view, see Derauf et al., 2009). This could help explain the volume reductions in cortical regions without high densities of monoaminergic receptors, and targeting of different neurotransmitter circuits by the two different drugs may explain why some brain regions are more or less reduced in volume in the two exposure groups.

Striatal volume reductions in children with prenatal MA exposure observed here are consistent with previous findings (Chang et al., 2004) and consistent with our predictions based on the known neurotoxicity of MA to dopamine-rich brain areas (Volkow et al., 2001), but volume reductions of striatal structures have also been observed in children with heavy prenatal alcohol exposure (Archibald et al., 2001). Striatal volume reductions observed in this sample were more severe in the MAA than ALC groups, suggesting that prenatal MA exposure or the combination of MA and alcohol exposure has a larger impact on the striatum than alcohol alone. Group $\times$ FSIQ interactions were significant in the left caudate, in which smaller volume was associated with lower FSIQ scores in the MAA group, and smaller volume was associated with higher FSIQ scores in the CON group. These findings, although preliminary, suggest that the damage to striatal structures resultant from prenatal MA exposure or to the combination of MA and alcohol exposure, and potential subsequent disturbances in maturational change within those structures is related to lower general intellectual functioning long after the perinatal insult(s) to the brain. This effect appears to be somewhat specific to the striatum because group $X$ score interactions were not significant in any other region evaluated, which makes sense given the prevalence of dopamine receptors and their vulnerability to MA toxicity. It should be noted, however, that these analyses were exploratory and should be interpreted with caution until replicated in independent samples. 

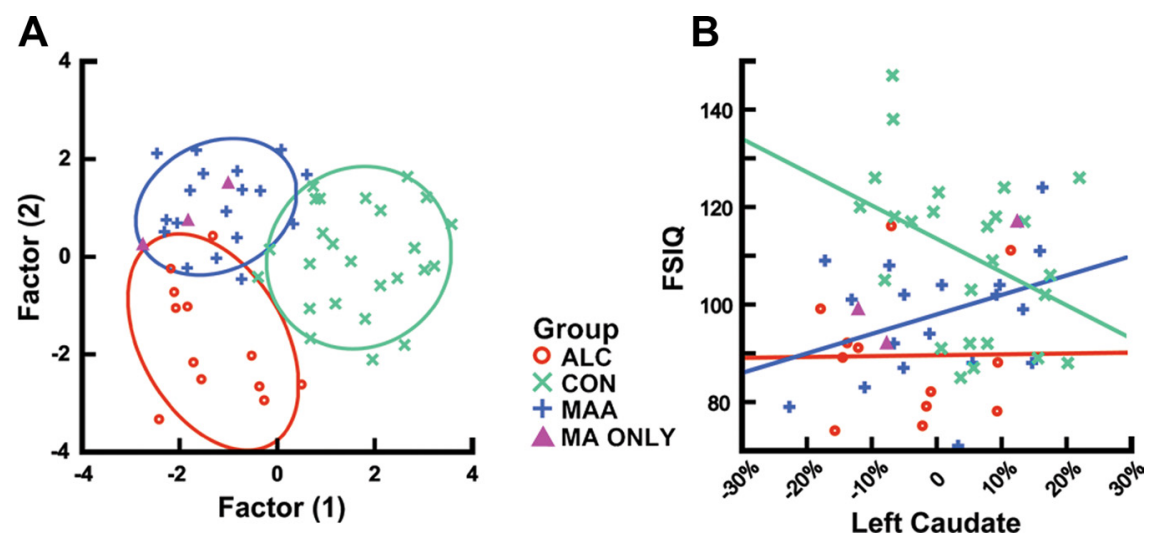

Figure 5. A, Factor analysis using Jacobian values for each of the 14 ROls (described in Table 1 and illustrated in Fig. 1) and full-scale intelligent quotient to predict group membership. $\boldsymbol{B}$, Group $\times$ score interactions in predicting left caudate volume, with volume measures presented as percentage change from the average MDT (i.e., $-10 \%$ is $10 \%$ smaller than the grand group average).

Volume reductions in left occipital brain regions, right occipital fusiform cortex, and bilateral dorsal parietal cortex were also more severe in the MAA than ALC subjects and, although unexpected, may be consistent with findings of decreased regional cerebral blood flow in left occipital cortices in adult MA abusers (Chang et al., 2002). Local volume increases were observed in both exposure groups relative to the unexposed controls, particularly in the limbic cortices of the anterior and posterior cingulate and inferior and medial temporal and bilateral perisylvian cortices. We have noted previously cortical thickness increases in lateral and medial temporal regions in independent samples of children with heavy prenatal alcohol exposure (Sowell et al., 2007), and others have noted relative volume sparing in medial temporal lobe structures in a similar population (Archibald et al., 2001). This is the first time volume increases in cingulate cortices in children with prenatal MA exposure have been reported, and, in a direct comparison of exposed groups, cingulate volume increases were more pronounced in the MAA than the ALC group (i.e., MAA > ALC). The anterior cingulate cortex is part of the attentional network shown to be deficient in children with prenatal MA exposure (Chang et al., 2004) and is associated with monitoring of control, conflict resolution, and decision making and generally connects sensory inputs with executive brain centers in generating motor outputs (for review, see Assadi et al., 2009). The anterior cingulate is also strongly interconnected with dopamine-rich striatal, medial temporal, and thalamic structures (for review, see Assadi et al., 2009), all shown to be dysmorphic in children with prenatal drug exposure. Dopamine receptors are most highly expressed in the striatum, but there is evidence that extrastriatal dopamine receptors exist in anterior cingulate and lateral temporal lobe structures (Okubo et al., 1999). Although we observe volume increases in extrastriatal limbic cortices in those with prenatal MA and/or alcohol exposure, a report in adult MA abusers show volume deficits in cingulate and medial temporal lobe structures (Thompson et al., 2004). Volume increases in cingulate and other interconnected limbic cortices may be compensatory for dysfunction in striatal and thalamic structures that are reduced in volume in children with MA and/or alcohol exposure. Alternatively, it is possible that volume increases are dysfunctional, resulting from lack of synaptic pruning and/or decreased myelination, cellular changes that are known to continue during childhood and adolescence, and are thought to result in more efficient cognitive processing (for review, see Sowell et al., 2003). Although correlations here between FSIQ and local volume in inferior temporal limbic regions and thalamus were significant when considered for all three groups combined, these correlations were primarily mediated by group membership. Additional study will be required to determine the cognitive significance of brain dysmorphology in children with prenatal exposure to drugs of abuse.

The difference in brain dysmorphology between the MAA and ALC groups highlights that structural brain imaging may yield more information about the integrity of brain networks than evaluations at the behavioral level alone. This statement is further supported by the results from discriminant function analyses presented here. Whether or not factor scores from the current sample could be used to predict new subjects has yet to be tested, but the estimate from jackknifed analyses of an overall $72 \%$ accurate classification is promising. Information from structural brain imaging may eventually be used diagnostically for children with cognitive or behavioral abnormalities but without well documented exposure histories. That different brain systems are more or less affected in these children with different drug exposure histories suggest that different interventions may be appropriate for cognitive and behavioral deficits depending on which brain systems are more affected.

There are limitations to this study that necessitate cautious interpretation of results. As with the vast majority of studies of prenatal exposure to drugs of abuse, quantities and frequencies of drug exposure are difficult to accurately recall years after the drug use and may be compounded by the stigma of admitting to drug use during pregnancy. When women receive methamphetamine from their partners, they may not actually know the dosage they ingest. Potential underreporting by biological mothers is of sufficient concern that reports from adoptive mothers (based on observation of biological mother's behavior or from social services reports) may have similar levels of validity to that of biological mothers. The majority of our MA-exposed participants also had concomitant alcohol exposure, and it is not clear that quantity and frequency of exposure were comparable between our ALC and MAA groups. The possibility that morphological differences observed in the MAA group is related to an interaction of methamphetamine with alcohol rather than to methamphetamine alone cannot be ruled out. Nonetheless, studying children whose exposure histories are representative of the communities from which they were drawn may be more ecologically valid than trying to find groups of children with "pure" exposures to only one drug.

Also of considerable concern is the concomitant nicotine exposure that is likely more prevalent in the exposed than nonexposed populations. Nicotine exposure is of particular concern because, in animal models, it has been shown to disrupt the timing of cell replication and differentiation, and such effects are longer lasting than effects from fetal cocaine exposure (Slotkin et al., 1998). In a recent survey of 191 prospectively identified MA exposed subjects, $\sim 80 \%$ were also exposed to nicotine (Della Grotta et al., 2009), and another recently published survey article, 55\% of women who reported drinking during pregnancy also admitted smoking (Aliyu et al., 2009). Thus, rates of coexposure to nicotine are high in general in those who abuse MA and/or 
alcohol, and similar rates are likely present in our samples, which would suggest that differences between the MAA and ALC groups are not likely attributable to differences in nicotine exposure. Finally, although mono-drug exposures are rare in humans, there is a vast animal literature suggesting that damage to the fetus occurs when alcohol (Sulik, 2005) or MA (Melo et al., 2008) are administered alone. Unfortunately, unavailability of nicotineexposure information from a significant proportion of our exposed subjects makes it difficult to evaluate whether the rate of nicotine exposure in the MAA and ALC groups differ. Finally, our sample sizes in the exposed groups were relatively small, but our sample sizes were sufficient to detect the group differences in brain morphology reported here. It remains possible, however, that insufficient power precluded us from detecting subthreshold differences between the ALC and MAA groups.

\section{References}

Aliyu MH, Wilson RE, Zoorob R, Brown K, Alio AP, Clayton H, Salihu HM (2009) Prenatal alcohol consumption and fetal growth restriction: potentiation effect by concomitant smoking. Nicotine Tob Res 11:36-43.

Archibald SL, Fennema-Notestine C, Gamst A, Riley EP, Mattson SN, Jernigan TL (2001) Brain dysmorphology in individuals with severe prenatal alcohol exposure. Dev Med Child Neurol 43:148-154.

Assadi SM, Yücel M, Pantelis C (2009) Dopamine modulates neural networks involved in effort-based decision-making. Neurosci Biobehav Rev 33:383-393.

Astley SJ (2004) Diagnostic guide for fetal alcohol spectrum disorders: the 4-Digit Diagnostic Code. Seattle, WA: The University of Washington.

Astley SJ, Aylward EH, Olson HC, Kerns K, Brooks A, Coggins TE, Davies J, Dorn S, Gendler B, Jirikowic T, Kraegel P, Maravilla K, Richards T (2009) Magnetic resonance imaging outcomes from a comprehensive magnetic resonance study of children with fetal alcohol spectrum disorders. Alcohol Clin Exp Res 33:1671-1689.

Autti-Rämö I, Autti T, Korkman M, Kettunen S, Salonen O, Valanne L (2002) MRI findings in children with school problems who had been exposed prenatally to alcohol. Dev Med Child Neurol 44:98-106.

Berman S, O’Neill J, Fears S, Bartzokis G, London ED (2008) Abuse of amphetamines and structural abnormalities in the brain. Ann N Y Acad Sci 1141:195-220.

Bookstein FL, Sampson PD, Streissguth AP, Connor PD (2001) Geometric morphometrics of corpus callosum and subcortical structures in the fetalalcohol-affected brain. Teratology 64:4-32.

Bookstein FL, Streissguth AP, Sampson PD, Connor PD, Barr HM (2002) Corpus callosum shape and neuropsychological deficits in adult males with heavy fetal alcohol exposure. Neuroimage 15:233-251.

Brun CC, Nicolson R, Leporé N, Chou YY, Vidal CN, DeVito TJ, Drost DJ, Williamson PC, Rajakumar N, Toga AW, Thompson PM (2009) Mapping brain abnormalities in boys with autism. Hum Brain Mapp 30:3887-3900.

Bullmore ET, Suckling J, Overmeyer S, Rabe-Hesketh S, Taylor E, Brammer MJ (1999) Global, voxel, and cluster tests, by theory and permutation, for a difference between two groups of structural MR images of the brain. IEEE Trans Med Imaging 18:32-42.

Chang L, Ernst T, Speck O, Patel H, DeSilva M, Leonido-Yee M, Miller EN (2002) Perfusion MRI and computerized cognitive test abnormalities in abstinent methamphetamine users. Psychiatry Res 114:65-79.

Chang L, Smith LM, LoPresti C, Yonekura ML, Kuo J, Walot I, Ernst T (2004) Smaller subcortical volumes and cognitive deficits in children with prenatal methamphetamine exposure. Psychiatry Res 132:95-106.

Chang L, Alicata D, Ernst T, Volkow N (2007) Structural and metabolic brain changes in the striatum associated with methamphetamine abuse. Addiction 102 [Suppl 1]:16-32.

Chang L, Cloak C, Jiang CS, Farnham S, Tokeshi B, Buchthal S, Hedemark B, Smith LM, Ernst T (2009) Altered neurometabolites and motor integration in children exposed to methamphetamine in utero. Neuroimage 48:391-397.

Che A, Cui J, Dinov ID (2009) SOCR analyses: implementation and demonstration of a new graphical statistics educational toolkit. J Stat Software 30:1-19.

Chiang MC, Reiss AL, Lee AD, Bellugi U, Galaburda AM, Korenberg JR, Mills
DL, Toga AW, Thompson PM (2007) 3D pattern of brain abnormalities in Williams syndrome visualized using tensor-based morphometry. Neuroimage 36:1096-1109.

Cloak CC, Ernst T, Fujii L, Hedemark B, Chang L (2009) Lower diffusion in white matter of children with prenatal methamphetamine exposure. Neurology 72:2068-2075.

Colliver JD, Kroutil LA, Dai L, Gfroerer JC (2006) Misuse of prescription drugs: data from the 2002, 2003, and 2004 National Surveys on Drug Use and Health. Rockville, MD: Substance Abuse and Mental Health Services Administration, Office of Applied Studies.

Della Grotta S, Lagasse LL, Arria AM, Derauf C, Grant P, Smith LM, Shah R, Huestis M, Liu J, Lester BM (2009) Patterns of methamphetamine use during pregnancy: results from the Infant Development, Environment, and Lifestyle (IDEAL) study. Matern Child Health J. Advance online publication. Retrieved Feb. 17, 2010. doi:10.1007/s10995-009-0491-0.

Derauf C, Kekatpure M, Neyzi N, Lester B, Kosofsky B (2009) Neuroimaging of children following prenatal drug exposure. Semin Cell Dev Biol 20:441-454.

Frost DO, Cadet JL (2000) Effects of methamphetamine-induced neurotoxicity on the development of neural circuitry: a hypothesis. Brain Res Brain Res Rev 34:103-118.

Glass LA, Ryan JJ, Bartels JM, Morris J (2008) Estimating WISC-IV indexes: proration versus linear scaling. J Clin Psychol 64:1175-1180.

Gogtay N, Lu A, Leow AD, Klunder AD, Lee AD, Chavez A, Greenstein D, Giedd JN, Toga AW, Rapoport JL, Thompson PM (2008) Threedimensional brain growth abnormalities in childhood-onset schizophrenia visualized by using tensor-based morphometry. Proc Natl Acad Sci U S A 105:15979-15984.

Hua X, Leow AD, Parikshak N, Lee S, Chiang MC, Toga AW, Jack CR Jr, Weiner MW, Thompson PM (2008a) Tensor-based morphometry as a neuroimaging biomarker for Alzheimer's disease: an MRI study of 676 AD, MCI, and normal subjects. Neuroimage 43:458-469.

Hua X, Leow AD, Lee S, Klunder AD, Toga AW, Lepore N, Chou YY, Brun C, Chiang MC, Barysheva M, Jack CR Jr, Bernstein MA, Britson PJ, Ward CP, Whitwell JL, Borowski B, Fleisher AS, Fox NC, Boyes RG, Barnes J, Harvey D, Kornak J, Schuff N, Boreta L, Alexander GE, Weiner MW, Thompson PM, Alzheimer's Disease Neuroimaging Initiative (2008b) 3D characterization of brain atrophy in Alzheimer's disease and mild cognitive impairment using tensor-based morphometry. Neuroimage 41:19-34.

Hua X, Leow AD, Levitt JG, Caplan R, Thompson PM, Toga AW (2009) Detecting brain growth patterns in normal children using tensor-based morphometry. Hum Brain Mapp 30:209-219.

Kochunov P, Lancaster JL, Thompson P, Woods R, Mazziotta J, Hardies J, Fox P (2001) Regional spatial normalization: toward an optimal target. J Comput Assist Tomogr 25:805-816.

Kochunov P, Lancaster J, Thompson P, Toga AW, Brewer P, Hardies J, Fox P (2002) An optimized individual target brain in the Talairach coordinate system. Neuroimage 17:922-927.

Lee AD, Leow AD, Lu A, Reiss AL, Hall S, Chiang MC, Toga AW, Thompson PM (2007) 3D pattern of brain abnormalities in Fragile X syndrome visualized using tensor-based morphometry. Neuroimage 34:924-938.

Leow A, Huang SC, Geng A, Becker J, Davis S, Toga A, Thompson P (2005) Inverse consistent mapping in $3 \mathrm{D}$ deformable image registration: its construction and statistical properties. Inf Process Med Imaging 19:493-503.

Leow AD, Yanovsky I, Chiang MC, Lee AD, Klunder AD, Lu A, Becker JT, Davis SW, Toga AW, Thompson PM (2007) Statistical properties of Jacobian maps and the realization of unbiased large-deformation nonlinear image registration. IEEE Trans Med Imaging 26:822-832.

Leow AD, Yanovsky I, Parikshak N, Hua X, Lee S, Toga AW, Jack CR Jr, Bernstein MA, Britson PJ, Gunter JL, Ward CP, Borowski B, Shaw LM, Trojanowski JQ, Fleisher AS, Harvey D, Kornak J, Schuff N, Alexander GE, Weiner MW, Thompson PM (2009) Alzheimer's disease neuroimaging initiative: a one-year follow up study using tensor-based morphometry correlating degenerative rates, biomarkers and cognition. Neuroimage 45:645-655.

Lu LH, Johnson A, O'Hare ED, Bookheimer SY, Smith LM, O'Connor MJ, Sowell ER (2009) Effects of prenatal methamphetamine exposure on verbal memory revealed with functional magnetic resonance imaging. J Dev Behav Pediatr 30:185-192.

McCann UD, Ricaurte GA (2004) Amphetamine neurotoxicity: accomplishments and remaining challenges. Neurosci Biobehav Rev 27:821-826. 
Melo P, Pinazo-Duran MD, Salgado-Borges J, Tavares MA (2008) Correlation of axon size and myelin occupancy in rats prenatally exposed to methamphetamine. Brain Res 1222:61-68.

O'Connor MJ, Frankel F, Paley B, Schonfeld AM, Carpenter E, Laugeson EA, Marquardt R (2006) A controlled social skills training for children with fetal alcohol spectrum disorders. J Consult Clin Psychol 74:639-648.

Okubo Y, Olsson H, Ito H, Lofti M, Suhara T, Halldin C, Farde L (1999) PET mapping of extrastriatal D2-like dopamine receptors in the human brain using an anatomic standardization technique and $\left[{ }^{11} \mathrm{C}\right]$ FLB 457 . Neuroimage 10:666-674.

Ratei R, Karawajew L, Lacombe F, Jagoda K, Del Poeta G, Kraan J, De Santiago M, Kappelmayer J, Björklund E, Ludwig WD, Gratama JW, Orfao A (2007) Discriminant function analysis as decision support system for the diagnosis of acute leukemia with a minimal four color screening panel and multiparameter flow cytometry immunophenotyping. Leukemia 21:1204-1211.

Riley EP, McGee CL (2005) Fetal alcohol spectrum disorders: an overview with emphasis on changes in brain and behavior. Exp Biol Med (Maywood) 230:357-365.

Shattuck DW, Leahy RM (2002) BrainSuite: an automated cortical surface identification tool. Med Image Anal 6:129-142.

Sled JG, Zijdenbos AP, Evans AC (1998) A nonparametric method for automatic correction of intensity nonuniformity in MRI data. IEEE Trans Med Imaging 17:87-97.

Slotkin TA (1998) Fetal nicotine or cocaine exposure: which one is worse? J Pharmacol Exp Ther 285:931-945.

Smith LM, Chang L, Yonekura ML, Grob C, Osborn D, Ernst T (2001) Brain proton magnetic resonance spectroscopy in children exposed to methamphetamine in utero. Neurology 57:255-260.

Smith LM, LaGasse LL, Derauf C, Grant P, Shah R, Arria A, Huestis M, Haning W, Strauss A, Della Grotta S, Liu J, Lester BM (2006) The infant development, environment, and lifestyle study: effects of prenatal methamphetamine exposure, polydrug exposure, and poverty on intrauterine growth. Pediatrics 118:1149-1156.

Sowell ER, Thompson PM, Mattson SN, Tessner KD, Jernigan TL, Riley EP, Toga AW (2002) Regional brain shape abnormalities persist into adolescence after heavy prenatal alcohol exposure. Cereb Cortex 12:856-865.

Sowell ER, Peterson BS, Thompson PM, Welcome SE, Henkenius AL, Toga AW (2003) Mapping cortical change across the human life span. Nat Neurosci 6:309-315.

Sowell ER, Mattson SN, Kan E, Thompson PM, Riley EP, Toga AW (2007) Abnormal cortical thickness and brain-behavior correlation patterns in individuals with heavy prenatal alcohol exposure. Cereb Cortex 18:136-144.

Sulik KK (2005) Genesis of alcohol-induced craniofacial dysmorphism. Exp Biol Med (Maywood) 230:366-375.

Thompson PM, Hayashi KM, Simon SL, Geaga JA, Hong MS, Sui Y, Lee JY, Toga AW, Ling W, London ED (2004) Structural abnormalities in the brains of human subjects who use methamphetamine. J Neurosci 24: $6028-6036$.

Volkow ND, Chang L, Wang GJ, Fowler JS, Leonido-Yee M, Franceschi D, Sedler MJ, Gatley SJ, Hitzemann R, Ding YS, Logan J, Wong C, Miller EN (2001) Association of dopamine transporter reduction with psychomotor impairment in methamphetamine abusers. Am J Psychiatry 158:377-382.

Wechsler D (2003) The Wechsler intelligence scale for children, Ed 4. San Antonio, TX: The Psychological Corporation.

Wells WM 3rd, Viola P, Atsumi H, Nakajima S, Kikinis R (1996) Multimodal volume registration by maximization of mutual information. Med Image Anal 1:35-51.

Yanovsky I, Leow AD, Lee S, Osher SJ, Thompson PM (2009) Comparing registration methods for mapping brain change using tensor-based morphometry. Med Image Anal 13:679-700. 